\title{
Correspondence
}

\section{Difficulties in interpreting patient-reported outcome measures in the absence of a gold standard: the meaning of Clinical Global Impressions scores in liaison psychiatry}

As a part of routine clinical care, the Ealing Liaison Psychiatry Service (ELPS) uses the Clinical Global Impressions (CGI) scale to assess patient-reported outcomes. We would like to share our findings and the challenges in interpreting CGI ratings.

CGI scales in psychiatry were initially used to assess efficacy in clinical drug trials ${ }^{1}$ and have since been adapted for use in liaison psychiatry as a part of a nationwide evaluation. The Framework for Routine Outcome Measurement in Liaison Psychiatry ${ }^{2}$ proposed that all liaison psychiatry services use CGI scales for consistent data collection and national reporting of outcomes, although there is no guidance on a standard to aim for.

Our methodology involved patients and their ELPS clinicians providing a CGI rating on whether the patient's mental health had improved, not changed or become worse after ELPS contact. This study looked at all 205 patients between January 2018 and November 2019 who had filled out a CGI questionnaire, and the following analyses were made:

(a) percentages of patients reporting changes in their mental health and potential reasons for this;

(b) concordance between patient and clinician ratings.

Fifty-nine per cent of patients reported an improvement in their mental health, although the reasons for this were unclear, given that the CGI questionnaire has no section for patients to justify their rating. A variety of factors may be involved, including having a focused consultation with a clinician, a decrease in symptom severity, and improvements in physical or social symptoms during the hospital stay, as these are often inextricably linked with mental health.

Forty per cent felt there was no change after ELPS intervention, and $1 \%$ (three patients) indicated feeling worse. Of the latter, two patients had to be admitted to an in-patient psychiatric unit, which could suggest that their lack of improvement was due to the severity of their mental health condition itself. There was 91\% concordance between patient and clinician ratings, suggesting that subjective ratings from patients may not be needed if clinicians' objective ratings are so closely tallied.

The CGI scale has been correlated with more timeconsuming rating scales used in psychiatry, ${ }^{3}$ and its advantages lie in its ease of understanding by both professionals and lay people, its ability to track progress across time and its swiftness of application. On reflection of our findings, we are unable to comment on our performance given the dearth of literature discussing what constitutes the gold standard. However, encouragingly, most patients reported improvement while only a very small minority reported deterioration, indicating that liaison psychiatry interventions are effective and largely beneficial.

By nature, the liaison psychiatry population comprises patients with both physical and mental health conditions, causing relative difficulty in teasing out which of the two issues is better manifested in the desirable outcome. In addition, the heterogeneity of the liaison psychiatry population makes it difficult to make direct comparisons of validity between different psychiatric conditions.

This simple service evaluation suggests that ELPS improves patients' well-being according to CGI scales. Nevertheless, wider-scale studies should be performed to elucidate how liaison psychiatry interventions are beneficial and to inform what the standard of care should be.

Rajalakshmi Valaiyapathi, West London NHS Trust, UK. Email: r.valaiyapathi@nhs.net; Kezia Smith, West London NHS Trust, UK. Email: kezia. smith2@nhs.net; Ksenia Marjanovic-Deverill, Liaison Psychiatry Consultant, West London NHS Trust, UK. Email: ksenia.marjanovic-deverill@westlondon. nhs.uk

1 Guy W. ECDEU Assessment Manual for Psychopharmacology Revised. US Department of Health, Education, and Welfare, 1976.

2. Faculty of Liaison Psychiatry. Framework for Routine Outcome Measurement in Liaison Psychiatry (FROM-LP), FR/LP/02. Royal College of Psychiatrists, 2015.

3 Busner J, Targum SD. The clinical global impressions scale: applying a research tool in clinical practice. Psychiatry 2007; 4(7): 28-37.

doi:10.1192/bjb.2021.47

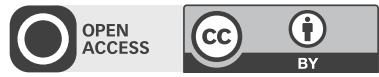

(c) The Author(s), 2021. Published by Cambridge University Press on behalf of the Royal College of Psychiatrists. . This is an Open Access article, distributed under the terms of the Creative Commons Attribution licence (http://creativecommons.org/licenses/by/4.0/), which permits unrestricted re-use, distribution, and reproduction in any medium, provided the original work is properly cited.

\section{Being positive on disability in medical education}

The world is a diverse place. The patients we treat are a diverse group. The same can't be said for the medical profession that represents them. I would argue that positivity regarding disabilities in medicine starts with us as educators. My favourite aspect of being a medical educator is what I as a teacher learn from my students. I, like most doctors and medical students, do not have a disability. However, I communicate with and advocate for people with intellectual disabilities, autism and learning difficulties such as dyspraxia and teach about these topics as routine practice to students and the wider multidisciplinary team. This is not just about reducing discrimination and promoting equality but also about appreciating the value that such people add to society.

General community estimates within the UK suggest the prevalence of disability in working age adults to be $19 \%$ in the $\mathrm{UK}^{1}$ yet in medical schools, it is estimated that only $4.1 \%$ of students have a disability. ${ }^{2}$ Even accounting for potential nondisclosure, these numbers show substantial underrepresentation. The British Medical Association recently published a report titled Disability in the Medical Profession ${ }^{3}$ that highlighted the paucity of doctors and medical students with disabilities 
who felt safe to disclose their condition or felt supported by their institution or colleagues. Despite widening participation programmes, medicine can still be discriminatory towards those with disabilities. It would not be unreasonable to suppose that such reluctance to seek support or have the need for this recognised could be extrapolated to our patients.

The term disability is broad, both in nature and degree. This is often poorly reflected in medical guidelines, whereby our lack of understanding can contribute to the pre-existing stigma. The title of the General Medical Council's Welcomed and Valued ${ }^{4}$ guidance for those with disabilities in the medical profession gives the impression of framing inclusivity in a positive light. However, the persistent use of terms like 'support', 'student needs' or educators and institutions applying their 'duties' means the focus is on reducing discrimination and making reasonable adjustments to allow for an assessment of competence. The presumption is that disability may be a threat to competence and to patient safety. In fact, disability may give lived experience that improves competency through empathy and understanding. We need to start focusing on what such students, doctors, educators and, crucially, patients can add, not simply what they need.

If we provide a safe space for those with lived experience of disabilities to share their experiences and the impact on their life, then each one of us can improve our understanding. Indeed, more doctors being open about their visible and invisible disabilities mean more positive role models for students. Widening participation can then move from just implementing antidiscrimination legislation to appreciating the value that diversity adds and the grassroots barriers to entering and thriving in the profession of medicine. Every medical educator can contribute by providing a safe space for disclosure and a willingness to learn and be educated by our patients and students.

Elliott Carthy, University of Oxford, UK. Email: elliott.carthy@psych.ox.ac.uk

\section{Declaration of interest}

None

1 Department of Work \& Pensions. Family Resources Survey 2018/19. 2020. Available from: https://www.gov.uk/government/statistics/familyresources-survey-financial-year-201819 [cited 27 Dec 2020].

2 Shrewsbury D. Disability and participation in the professions: examples from higher and medical education. Disabil Soc 2015; 30(1): 87-100

3 British Medical Association. Disability in the Medical Profession. Available from: https://www.bma.org.uk/advice-and-support/nhs-delivery-andworkforce/workforce/disability-in-the-medical-profession [cited 27 Dec 2020].

4 General Medical Council. Welcomed and Valued: Supporting Disabled Learners in Medical Education and Training. Available from: https:// www.gmc-uk.org/education/standards-guidance-and-curricula/guidance/welcomed-and-valued [cited 27 Dec 2020].

doi:10.1192/bjb.2021.48
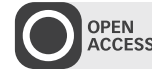

OPEN

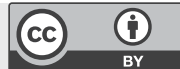

(c) The Author(s), 2021. Published by Cambridge University Press on behalf of the Royal College of Psychiatrists. . This is an Open Access article, distributed under the terms of the Creative Commons Attribution licence (http://creativecommons.org/licenses/by/4.0/), which permits unrestricted re-use, distribution, and reproduction in any medium, provided the original work is properly cited.

\section{More research needed into long-term medical student mental health during COVID-19 pandemic and beyond}

The mental health of healthcare professionals has been significantly affected by the COVID-19 pandemic. ${ }^{1}$ We believe that more research focusing specifically on the long-term mental health of medical students is required. Medical students are an at-risk group, with a greater incidence of anxiety ${ }^{2}$ than the general population, and a higher prevalence of suicidal ideation than physicians and nurses. ${ }^{3}$ The risk is further compounded by being young, ${ }^{1}$ having higher awareness of disease severity, and possessing common personality traits such as maladaptive perfectionism and difficulty adapting to disruption in routine. ${ }^{2}$

Medical students often face a sense of duty to volunteer in hospitals in times of global health emergencies such as the current pandemic, which can bring particular emotional challenges. The subsequent higher risk of transmitting COVID-19 may cause increased social isolation. Uncertainty around medical education due to cancelled exams and placements also increases stress. ${ }^{4}$

Although a recent meta-analysis showed no difference in anxiety during COVID-19 in medical students, ${ }^{2}$ isolated studies worldwide have shown increased anxiety in those whose loved ones had contracted COVID-19 and who had interacted with COVID-19 patients. ${ }^{4}$

Higher levels of baseline stress and depression are negative predictors of poor mental health, ${ }^{1}$ so more research must be done to identify those especially at risk. Those with preexisting mental health issues have reported decreased access to the usual support services during the pandemic. ${ }^{1}$ To our knowledge, there is no available literature exploring the impact of COVID-19 on medical students with pre-existing mental illness. Experiences in previous pandemics such as SARS show that long-term mental health issues such as alcohol misuse and post-traumatic stress symptoms can persist for several years in healthcare workers who have been quarantined or have worked in high-risk areas. ${ }^{5}$

The majority of current studies of mental health in COVID-19 have been cross-sectional, and few have focused on medical students. Longitudinal, large-scale, multi-country studies focusing on medical students of all age groups and investigating more variables, such as pre-existing mental illness, are required to identify those most at risk and the long-term effects on this population. The results of these studies could be used to improve future implementation of targeted medical student-specific mental healthcare interventions. ${ }^{1}$

Anusha Mahesh Prabhu, Imperial College London, UK. Email: anusha. prabhu@hotmail.com; Intishar Rashad, Armed Forces Medical College, Dhaka Cantonment, Dhaka-1206, Bangladesh. Email: intisharrashad@gmail. com

\section{Acknowledgments}

Both authors have contributed equally in preparing this article.

\section{Declaration of interest}

None 